\title{
Development of Isotope-Microscopy and Implications for Cosmochemistry
}

\author{
H. Yurimoto, * and K. Nagashima* \\ * Department of Earth and Planetary Sciences, Tokyo Institute of Technology, Ookayama, Meguro, \\ Tokyo 152-8551 Japan
}

Isotopes are useful tracer in cosmo- and geo-chemistry to determine origin and circulation of elements in nature. Environmental changes shift isotope ratios of individual chemical phases and the variations often fixed in growing minerals. Therefore decoding of isotopic zonings of minerals is useful to study historical environmental variations. Because minerals are typically $\mathrm{mm}$-sizes, it is necessary to use microscopy to image such zoning. However, isotope-microscopy had not been established for this purpose because isotope variations in nature are very small (usually less than \% order). Recently several technical developments of secondary ion mass spectrometry (SIMS) are tried to achieve this analysis.

One state-of-the-art approach for the isotope-microscopy is use of scanning of focused ion beam. About $100 \mathrm{~nm}$ lateral resolution with a precision of 5 to $10 \%$ level for oxy gen isotope ratios on a $\sim 10 \mu \mathrm{m} \times 10 \mu \mathrm{m}$ sample field has been achieved by a Cameca Nano-SIMS apparatus [1]. The precision and the analyzed field should be complementary in a limited analytical time because secondary ion intensities per pixel are less than Mega-cps due to the fine focused primary beam, which determine the lateral resolution. Another approach is use of stigmatic ion optics with twodimensional detector. About $1 \mu \mathrm{m}$ lateral resolution with a precision of 0.5 to $1 \%$ level for oxygen isotope ratios on a $\sim 70 \times 70 \mu \mathrm{m}$ sample field has been achieved by a Cameca ims-1270 apparatus attached with SCAPS detector [2]. In the latter case, the lateral resolution and the precision are limited by aberrations of the optics and primary beam density of the analyzed field, respectively. The analyzed field is limited by optics configuration. Therefore, the scanning method has advantages in high lateral resolution isotope-microscopy of small area, whereas the stigmatic method is in high precision isotope-microscopy of wide area. In this talk we introduce a stigmatic isotope-microscopy developed in our laboratory.

Since stigmatic ion optics was realized [3], two-dimensional ion detector for quantitative imaging is highly demanded. SCAPS is a high-efficiency stacked CMOS-type active pixel sensor, which has several advantages over conventional two-dimensional detection systems including wide dynamic range, no insensitive period, direct detection of charged particles and constant ion-detection sensitivities from $\mathrm{H}$ to $\mathrm{U}$ [4]. Hence SCAPS can measure high ion flux with an accuracy of the statistical error and with detection limit corresponding to 3 incident ions [5]. The detector is equipped with Cameca ims-1270 ion microscope as an isotope microscope system [2]. Using the isotope microscope system, presolar silicate grains have been firstly identified in meteorites by insitu analy sis (Fig. 1) and oxy gen isotopic exchange process in the early solar system has been firstly visualized from the meteorite (Fig. 2).

\section{References}

[1] S. Messenger et al., Science. 300 (2003) 105.

[2] H. Yurimoto et al., Appl. Surf. Sci. 2003-204 (2003) 793. 
[3] R. Castaing and G. Slodzian, J. Microsc. 1, (1962) 395.

[4] K. Nagashima et al., Surf. Interf. Anal. 30 (2001) 131.

[5] I. Takayanagi et al., IEEE Trans. Electron Dev. 50 (2003) 70.

[6] K. Nagashima et al., Nature, 428 (2004) 921.

[7] K. Nagashima et al., http://www.lpi.usra.edu/meetings/chondrites2004/pdf/9072.pdf (2004)

[8] This research was partly supported by Monka-sho grants.

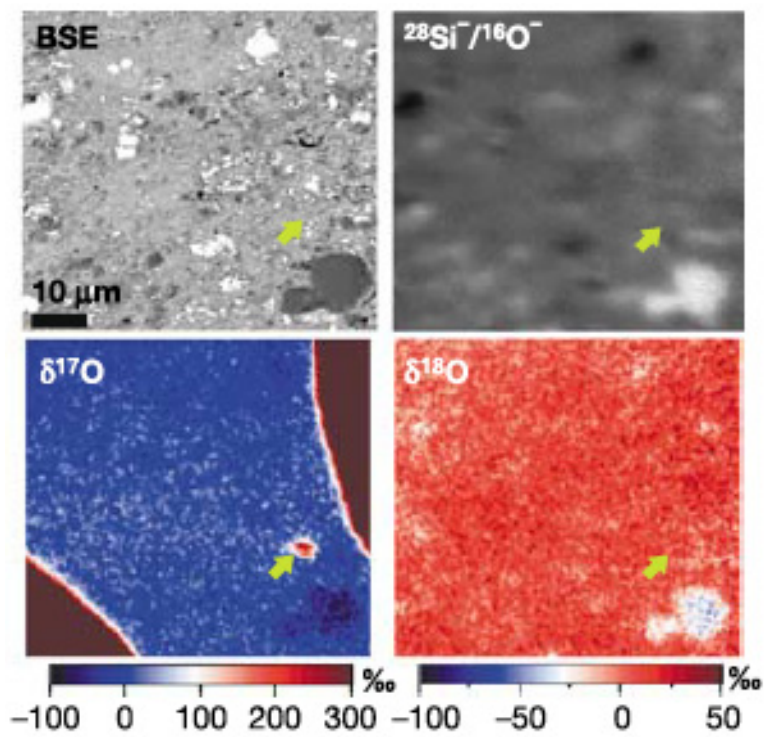

FIG. 1. Corresponding images of backscattered electrons, secondary ion ratio $\left({ }^{28} \mathrm{Si} /{ }^{16} \mathrm{O}\right)$, and oxy gen isotopograph $\left(\delta^{17} \mathrm{O}\right.$ and $\left.\delta^{18} \mathrm{O}\right)$ of matrices of Acfer 094 . The lateral resolution of the isotopographs is $\sim 1 \mu \mathrm{m}$. From [6].

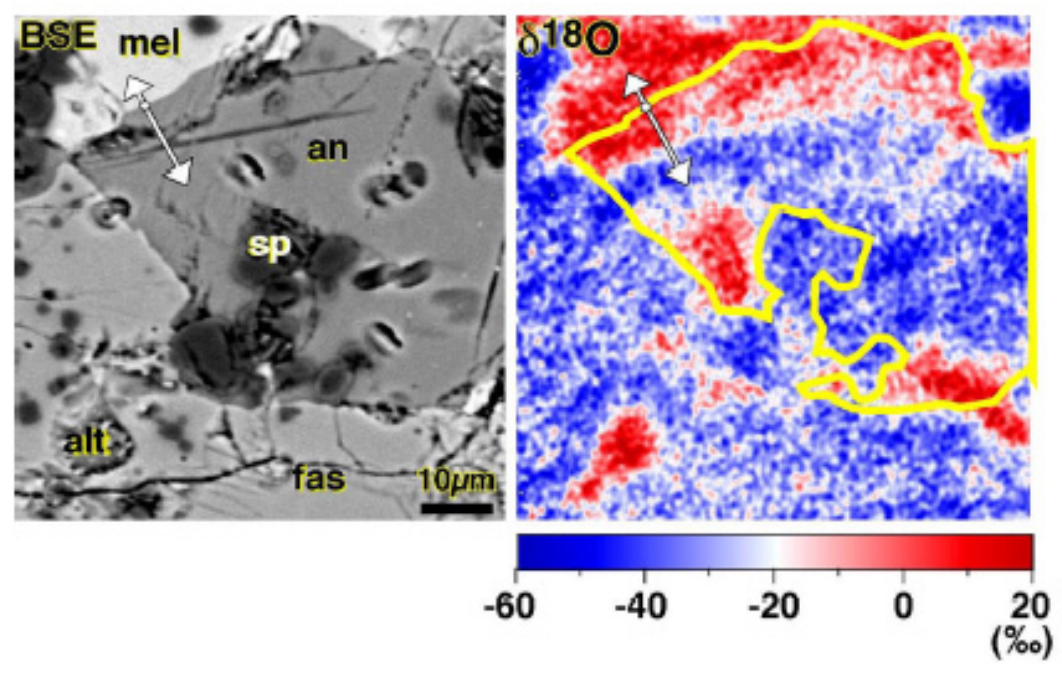

FIG. 2. Corresponding images of backscattered electrons and oxy gen isotopograph $\left(\delta^{18} \mathrm{O}\right)$ of an area containing anorthite \#B. Yellow curves indicate outline of anorthite \#B grain. Craters of SIMS analy ses are observed in the backscattered electron image. Abbreviations: $\mathrm{sp}=$ spinel, mel = meliilite, an $=$ anorthite, fas $=$ fassaite, and alt $=$ alteration products. From [7]. 